\section{Desigualdades na realização do exame clínico de mama em São Leopoldo, Rio Grande do Sul, Brasil}

\author{
Inequalities in clinical breast examination in \\ São Leopoldo, Rio Grande do Sul, Brazil
}

\begin{abstract}
This cross-sectional population-based survey focused on the coverage of clinical breast examination (CBE) in women 20 to 60 years of age in São Leopoldo, Rio Grande do Sul State, Brazil, and described the factors associated with lack of CBE. The outcome was defined as having received a $C B E$ by a physician during the year prior to the interview. Two different multivariate models were analyzed. Among the 1,026 women included in the sample, 556 (54.2\%; 95\%CI: 51.1-57.2) had received CBEs. Multivariate analysis including the entire sample of women showed that low socioeconomic status (SES) was the only variable associated with not having a CBE. In the other multivariate model (only women who had consulted physicians in the previous year, with or without CBE), a statistically significant effect was found for both SES and skin color. Thus, low SES was significantly associated with lack of CBE in both models. However, in the model limited to women with physician consultations in the previous year, skin color was the main factor for receiving $C B E$, suggesting that once differences in initial access to the health service were overcome, white women had a higher probability of receiving a clinical breast examination as part of their consultation.
\end{abstract}

Physical Examination; Breast; Women's Health; Health Inequalities

\author{
Juvenal Soares Dias-da-Costa 1,2 \\ Maria Teresa Anselmo Olinto 2 \\ Diego Bassani 2 \\ Carmem Regina Estivalete Marchionatti 2 \\ Fernanda Souza de Bairros ${ }^{2}$ \\ Maria Luísa Pereira de Oliveira ${ }^{2}$ \\ Milton Hartmann 2 \\ Roseclér Machado Gabardo 2 \\ Vivian Bennemann 2 \\ Marcos Pascoal Pattussi 2
}

\section{Introdução}

Segundo o Ministério da Saúde, o câncer de mama é a maior causa de óbitos por neoplasias na população feminina no Brasil. Em 2002, atingiu uma taxa de mortalidade de 10,2 por $100 \mathrm{mil}$ mulheres na faixa etária de 40 a 69 anos 1 . Para o mesmo período, no Estado do Rio Grande do Sul, a neoplasia maligna da mama foi a principal causa de óbito entre todas as formas de câncer que acometeram as mulheres ${ }^{2}$. Em Porto Alegre, a taxa de incidência de câncer de mama ajustada por idade foi a terceira entre as capitais brasileiras, atingindo 66,5 por 100 mil mulheres 3. Em São Leopoldo, durante o ano 2000, o câncer de mama foi a segunda neoplasia mais importante entre as mulheres de 20 a 59 anos de idade 4 .

Estudos têm mostrado que exame clínico de mama e mamografia são importantes métodos para a detecção precoce do câncer de mama 5,6. Sendo que no rastreamento para prevenção secundária de câncer de mama, o exame clínico de mama combinado com a mamografia pode aumentar a sensibilidade ${ }^{7}$.

Assim, o Programa de Controle do Câncer de Mama, proposto pelo Instituto Nacional de Câncer (INCA) em seu documento de consenso, tem preconizado que todas as mulheres a partir de 40 anos devem ser submetidas ao exame clínico de mama anualmente como forma de detecção precoce de lesões sugestivas de câncer. Aconse- 
lha, também, que as mulheres a partir de 35 anos que apresentarem determinados fatores de risco devem ser submetidas a esse exame. O programa preconiza, como método coadjuvante para o diagnóstico, a realização de mamografia anual entre as mulheres de 50 a 69 anos, e mamografia anual para mulheres a partir de 40 anos com risco aumentado ${ }^{8}$.

Contudo, deve-se ressaltar que o teto físico para atender às exigências do consenso é insuficiente em São Leopoldo, Rio Grande do Sul. Em 2005, o município apresentava uma população feminina de 50 a 69 anos de 14.011 mulheres; foram aprovados pelo Sistema Único de Saúde (SUS) em 2004 apenas 800 exames de mamografia. Essa diferença revela a defasagem entre as necessidades e a oferta de teto físico estabelecido na Programação Pactuada Integrada e reforça a necessidade da realização do exame clínico de mama.

Portanto, o presente trabalho pretende estabelecer o nível de cobertura de exame clínico de mamas entre as mulheres de 20 a 60 anos residentes em São Leopoldo, e descrever as características associadas a não-realização do exame.

\section{Materiais e métodos}

A Universidade do Vale do Rio dos Sinos (UNISINOS), por meio de um grupo de pesquisadores, elaborou um projeto de pesquisa intitulado Condições de Saúde das Mulheres: Estudo de Base Populacional na Região do Vale do Rio dos Sinos. Essa investigação foi um estudo transversal de base populacional que incluiu mulheres de 20 a 60 anos residentes na zona urbana da cidade de São Leopoldo. O projeto foi submetido e aprovado pelo Comitê de Ética e Pesquisa da Faculdade de Medicina da Universidade Federal de Pelotas.

A amostra inicial foi estimada levando-se em consideração alguns parâmetros incluídos no estudo, nível de confiança de 95\%, poder de $80 \%$, razão de não-expostos: expostos, estabelecida em 1:3 (baseada na distribuição de classe social encontrada em Pelotas, Rio Grande do Sul, ou seja, 30,7\% das mulheres inseridas nas classes A e B e quase $70 \%$ nas demais categorias ${ }^{9}$ ), razão de prevalência de 2,0.

Adicionou-se ao tamanho da amostra mais $10 \%$ de possíveis perdas e recusas e $15 \%$ para controlar fatores de confusão na análise dos dados.

Foi realizada uma amostra por conglomerados, sendo sorteados quarenta setores censitários entre os 270 existentes na zona urbana da cidade de São Leopoldo. Cada um desses setores teve o primeiro quarteirão sorteado juntamente com o ponto de partida, garantindo-se aleatorie- dade na coleta de dados. Dessa forma, para completar a população necessária foram visitados 36 domicílios em cada setor.

Foram utilizados questionários padronizados, pré-codificados e pré-testados. O grupo de entrevistadoras foi composto por acadêmicas de cursos na área da saúde da UNISINOS, treinadas para a aplicação do instrumento. Também foi realizado teste piloto para treinamento das entrevistadoras e os dados foram coletados entre março e dezembro de 2003.

O controle de qualidade foi realizado em uma amostra aleatória de $10 \%$ das pessoas incluídas no estudo, com o intuito de avaliar a qualidade da coleta de dados. O questionário de controle foi semelhante ao do estudo, incluindo variáveis que não se alteravam em curto espaço de tempo.

Após a finalização da coleta dos dados foram encontradas 1.084 mulheres de 20 a 60 anos, destas, $58(5,3 \%)$ foram classificadas como perdas e recusas, sendo incluídas no estudo 1.026 pessoas entrevistadas.

O desfecho foi definido como realização de exame clínico de mamas por médico no ano que antecedeu a entrevista.

As variáveis demográficas analisadas foram idades agrupadas (20 a 29; 30 a 39; 40 a 49; 50 a 60 anos), cor da pele observada (brancas; não-brancas) e estado civil (casada e em união; outros).

Foram analisadas como variáveis sócio-econômicas: classe econômica segundo a Associação Nacional de Empresas de Pesquisa (ANEP) 10, escolaridade, e se as mulheres estavam trabalhando no momento da entrevista. A classe econômica da ANEP foi construída a partir da posse de determinados bens materiais, escolaridade do chefe da família e número de empregados na família. A escolaridade das mulheres foi coletada como anos completos de estudo e categorizada de forma agrupada (0 a 4; 5 a 7; 8 a 11; 12 a 14; 15 ou mais).

Foram analisadas algumas variáveis relativas a morbidades, tais como: hipertensão arterial sistêmica, diabetes e obesidade. Definiu-se hipertensão arterial sistêmica por pressão arterial $\geq 140 / 90 \mathrm{mmHg}$ a partir da média de duas medidas (início e fim da aplicação do questionário individual) ou o uso atual de medicação antihipertensiva 11. A medida de tensão arterial foi coletada com esfigmomanômetro aneróide, com paciente apresentando membro superior direito despido. Através das medidas de peso e altura as mulheres foram classificadas quanto ao estado nutricional segundo o índice de massa corporal. O ponto de corte para definir sobrepeso foi IMC $\geq 25 \mathrm{~kg} / \mathrm{m}^{2} 12$. As mulheres consideradas com diabetes mellitus foram aquelas que referiram diagnóstico da doença realizado por médico. 
As variáveis consideradas como ginecológicas foram gestações prévias (nenhuma; de uma a três vezes; quatro ou mais), número de filhos (nenhum; 1 a 3; 4 ou mais) e história familiar de câncer de mama em parentes de primeiro e segundo graus.

Foram investigados alguns hábitos de vida, tais como: consumo abusivo de álcool e hábito de fumar (não-fumante; ex-fumante; fumante). O consumo de álcool foi avaliado mediante tipo, freqüência e quantidade. $O$ ponto de corte utilizado para definir consumo abusivo foi de $30 \mathrm{~g} /$ dia de etanol ou mais 13. Considerou-se como fumante o indivíduo que faz uso de cigarro diariamente, independente da quantidade.

A utilização de serviços de saúde foi analisada avaliando-se o número anual de consultas médicas (5 ou mais; de 3 a 4; de 1 a 2) e tipo de serviços de saúde (públicos; seguro privado; privados).

A entrada dos dados foi realizada com dupla entrada, havendo comparação entre elas na busca de diminuir erros de digitação, sendo utilizado o programa Epi Info 6.0 (Centers for Disease Control and Prevention, Atlanta, Estados Unidos).
As análises univariada e bivariada dos dados foram realizadas utilizando-se os programas SPSS (SPSS Inc., Chicago, Estados Unidos) e Stata (Stata Corp., College Station, Estados Unidos). Nessa fase, foram calculadas as razões de prevalência, os intervalos de confiança e testes estatísticos: qui-quadrado e tendência linear para as variáveis ordinais. $\mathrm{O}$ efeito do delineamento amostral para o desfecho exame de mamas no ano que antecedeu a entrevista entre as mulheres foi de 0,93 e as análises não necessitaram de correção.

Para controle dos fatores de confusão, a análise multivariada foi obtida através de regressão de Poisson, sendo que as variáveis foram incluídas de acordo com modelo hierarquizado definido na Figura 1 14. Foram utilizados dois modelos de análise: um incluindo todas as mulheres da amostra e outro excluindo as mulheres que não se consultaram com medico no último ano. Portanto, as variáveis de uso de serviços de saúde ingressaram no modelo de análise apenas para as mulheres que se consultaram com médico no ano que antecedeu a entrevista. Na modelagem hierarquizada por níveis foram mantidas as va-

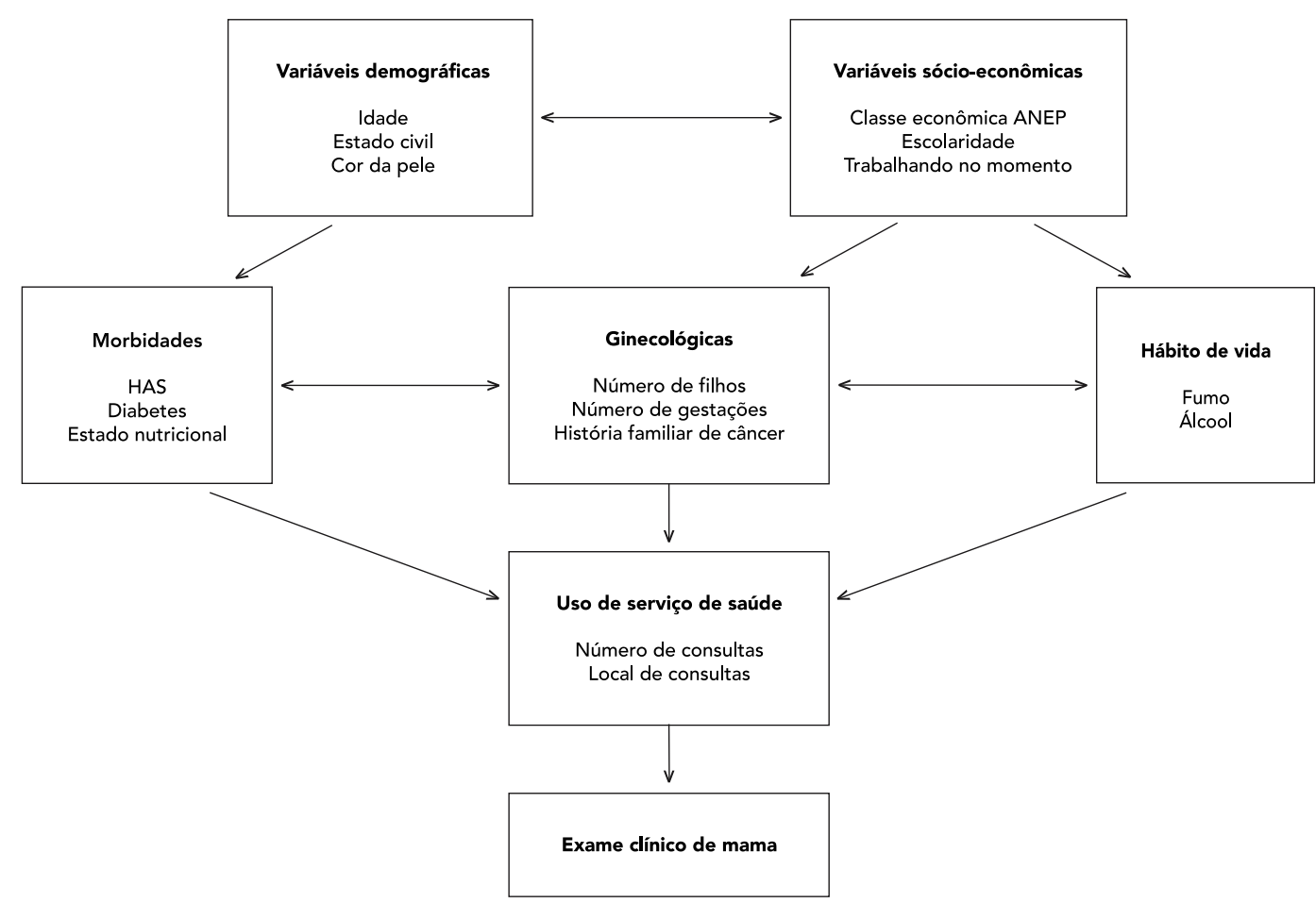

ANEP: Associação Nacional de Empresas de Pesquisa; HAS: hipertensão arterial sistêmica. 
riáveis que atingiram valor de $\mathrm{p} \leq 0,2$ no teste da razão de verossimilhança. Os modelos foram obtidos através de eliminação retrógrada.

\section{Resultados}

Entre as 1.026 mulheres incluídas na amostra, 556 (54,2\%; IC95\%: 51,1-57,2) foram submetidas ao exame clínico de mamas no ano que antecedeu a entrevista.

Em relação às variáveis demográficas, $51 \%$ das mulheres tinham menos de 39 anos, 83,8\% eram brancas e $64,2 \%$ da amostra eram casadas ou viviam em união estável (Tabela 1).

Quanto às características sócio-econômicas, observou-se que cerca de $67 \%$ das entrevistadas pertenciam às classes sociais $\mathrm{C}, \mathrm{D}$ e E, $44,4 \%$ possuíam sete ou menos anos de estudo e mais de $57 \%$ das mulheres referiram trabalhar fora (Tabela 1).

Verificou-se que $12,7 \%$ das mulheres eram hipertensas, $3,7 \%$ referiram diabetes e aproximadamente $50 \%$ apresentavam obesidade (Tabela 1 ).

Entre as variáveis ginecológicas, constatouse que mais de $80 \%$ das mulheres referiram no mínimo uma gestação, 64,5\% possuíam de 1 a 3 filhos e 12,4\% tinham história familiar de câncer de mama (Tabela 1).

Com relação aos hábitos de vida, 5,8\% das entrevistadas consumiam mais de $30 \mathrm{~g}$ de etanol por dia e $59 \%$ nunca fumaram (Tabela 1 ).

Entre as 887 mulheres que procuraram serviços de saúde, mais de $40 \%$ se consultaram com médico no mínimo cinco vezes no último ano e predominou a procura pelos seguros privados (Tabela 1).

$\mathrm{Na}$ análise bivariada, através dos intervalos de confiança e dos testes estatísticos, demonstrou-se que as prevalências da não-realização do exame diminuíram conforme aumentou a idade das mulheres. Entretanto, os intervalos de confiança e o teste estatístico mostraram diferenças limítrofes entre as categorias. As mulheres nãobrancas realizaram menos exames que as mulheres brancas e as mulheres casadas/em união foram mais examinadas (Tabela 1 ).

A maior prevalência de não-realização de exames ocorreu nas mulheres inseridas nas classes D e E. Observa-se que $60 \%$ das mulheres dessas classes não foram examinadas. Quanto menor a escolaridade, maior a probabilidade das mulheres não terem suas mamas examinadas. (Tabela 1).

Quanto maior o número de gestações e de filhos, maior a prevalência de não-realização do exame de mama. Assim, mulheres que tiveram quatro ou mais gestações apresentaram proba- bilidade $37 \%$ maior de não realizar exames de mama. Aquelas com quatro ou mais filhos foram também menos examinadas do que as mulheres com nenhum filho. Os resultados mostraram que aquelas com história familiar de câncer de mama possuíam probabilidade $33 \%$ menor de não realizar o exame.

Observou-se que mulheres fumantes possuíam uma probabilidade $20 \%$ maior de não realizar exames de mama. As mulheres que consumiam $30 \mathrm{~g}$ ou mais de etanol por dia apresentaram $21 \%$ mais probabilidade de não realizarem exames de mama, embora o limite inferior do intervalo de confiança tenha sido de 0,96 (Tabela 1).

Em relação à utilização de serviços de saúde, constatou-se que quanto menor o número de consultas, maior a probabilidade de não-realização de exame clínico de mama. As entrevistadas que utilizaram serviço de saúde privado tiveram maior probabilidade de realizar exames de mama quando comparadas com aquelas que referiram consultas no sistema público (Tabela 1).

Trabalho no momento da entrevista, hipertensão, diabetes e obesidade não apresentaram associações estatisticamente significativas com exame de mama ( $p>0,2)$.

A análise multivariada incluindo todas as mulheres demonstrou que somente a variável classe econômica apresentava associação estatisticamente significativa para a não-realização de exame médico de mama. As mulheres inseridas nas classes $\mathrm{C}, \mathrm{D}$ e $\mathrm{E}$ apresentaram menor probabilidade de realizarem exame de mama (Tabela 2).

$\mathrm{Na}$ análise multivariada que excluiu do modelo as mulheres que não se consultaram no último ano, constatou-se efeito significativo para classe econômica e cor da pele. Verificou-se aumento na medida de efeito para as mulheres de classes C, D e E de não terem suas mamas examinadas, quando comparadas com as mulheres da classe A. A variável cor da pele apresentou significância estatística, sendo que as mulheres brancas tiveram maior probabilidade de suas mamas serem examinadas (Tabela 3 ).

\section{Discussão}

Os estudos transversais podem fornecer valiosas informações para a organização e gestão de sistemas de saúde, desde que representem a população de referência ${ }^{15}$. O presente estudo incluindo amostra da população feminina de São Leopoldo apresentou em cada faixa etária a mesma distribuição proporcional verificada no Censo Demográfico de 2000, caracterizando sua representatividade. 
Distribuição da amostra e características associadas à ausência de exame médico de mama. São Leopoldo, Rio Grande do Sul, Brasil, 2003.

\begin{tabular}{|c|c|c|c|c|c|c|c|}
\hline \multirow[t]{2}{*}{ Variável } & \multirow[t]{2}{*}{$\mathbf{n}$} & \multirow[t]{2}{*}{$\%$} & \multicolumn{2}{|c|}{ Prevalência de não-exame (\%) } & \multirow[t]{2}{*}{ RP } & \multirow[t]{2}{*}{ IC95\% } & \multirow[t]{2}{*}{ p valor } \\
\hline & & & $\mathbf{n}$ & $\%$ & & & \\
\hline Idade (anos) & & & & & & & 0,087 \\
\hline $20-29$ & 283 & 27,6 & 146 & 51,6 & 1,00 & & \\
\hline $30-39$ & 255 & 24,9 & 118 & 46,3 & 0,90 & $0,75-1,07$ & \\
\hline $40-49$ & 302 & 24,4 & 128 & 42,4 & 0,82 & $0,69-0,98$ & \\
\hline $50-60$ & 186 & 18,1 & 78 & 41,9 & 0,81 & $0,66-1,00$ & \\
\hline Cor da pele & & & & & & & $<0,001$ \\
\hline Branca & 860 & 83,8 & 375 & 43,6 & 1,00 & & \\
\hline Não-branca & 166 & 16,2 & 95 & 57,2 & 1,31 & $1,13-1,53$ & \\
\hline Estado civil & & & & & & & 0,03 \\
\hline Casada/Em união & 659 & 64,2 & 286 & 43,4 & 1,00 & & \\
\hline Outros & 367 & 35,8 & 184 & 50,1 & 1,15 & $1,01-1,32$ & \\
\hline Classe social & & & & & & & $<0,001$ \\
\hline A & 74 & 7,2 & 12 & 16,2 & 1,00 & & \\
\hline$B$ & 265 & 25,9 & 80 & 30,2 & 1,86 & $1,07-3,22$ & \\
\hline C & 402 & 39,3 & 205 & 51,0 & 3,14 & $1,86-5,33$ & \\
\hline$D$ e $E$ & 286 & 27,6 & 171 & 60,6 & 3,74 & $2,21-6,33$ & \\
\hline Escolaridade (anos) & & & & & & & $<0,001$ \\
\hline 15 ou + & 117 & 11,4 & 28 & 23,9 & 1,00 & & \\
\hline $12-14$ & 285 & 27,8 & 105 & 36,8 & 1,54 & $1,08-2,20$ & \\
\hline $8-11$ & 169 & 16,5 & 91 & 53,8 & 2,25 & $1,58-3,20$ & \\
\hline $5-7$ & 247 & 24,1 & 130 & 52,6 & 2,20 & $1,56-3,10$ & \\
\hline $0-4$ & 208 & 20,3 & 116 & 55,8 & 2,33 & $1,65-3,29$ & \\
\hline Trabalhando no momento & & & & & & & 0,27 \\
\hline Sim & 591 & 57,6 & 262 & 44,3 & 1,00 & & \\
\hline Não & 435 & 42,4 & 208 & 47,8 & 1,08 & $0,94-1,23$ & \\
\hline Hipertensão & & & & & & & 0,41 \\
\hline Não & 892 & 87,3 & 413 & 46,3 & 1,00 & & \\
\hline Sim & 130 & 12,7 & 55 & 42,3 & 0,91 & $0,74-1,13$ & \\
\hline Diabetes & & & & & & & 0,58 \\
\hline Não & 988 & 96,3 & 451 & 45,6 & 1,00 & & \\
\hline Sim & 38 & 3,7 & 19 & 50,0 & 1,10 & $0,79-1,52$ & \\
\hline Obesidade & & & & & & & 0,68 \\
\hline Não-obeso & 506 & 50,8 & 230 & 45,5 & 1,00 & & \\
\hline Obeso & 490 & 49,2 & 229 & 46,7 & 1,03 & $0,90-1,18$ & \\
\hline Gestações & & & & & & & $<0,001$ \\
\hline Nenhuma & 204 & 19,9 & 86 & 42,2 & 1,00 & & \\
\hline $1-3$ & 631 & 61,5 & 274 & 43,4 & 1,03 & $0,86-1,24$ & \\
\hline 4 ou + & 191 & 18,6 & 110 & 57,6 & 1,37 & $1,12-1,67$ & \\
\hline Filhos & & & & & & & $<0,001$ \\
\hline Nenhum & 223 & 21,7 & 95 & 42,6 & 1,00 & & \\
\hline $1-3$ & 662 & 64,5 & 288 & 43,5 & 1,02 & $0,86-1,22$ & \\
\hline 4 ou + & 141 & 13,7 & 87 & 61,7 & 1,45 & $1,18-1,77$ & \\
\hline História familiar de câncer de mama & & & & & & & 0,03 \\
\hline Não & 899 & 87,6 & 424 & 47,2 & 1,00 & & \\
\hline Sim & 127 & 12,4 & 46 & 36,2 & 0,77 & $0,60-0,98$ & \\
\hline Uso de álcool (g/dia) & & & & & & & 0,11 \\
\hline$<30$ & 966 & 94,2 & 437 & 45,2 & 1,00 & & \\
\hline$\geq 30$ & 60 & 5,8 & 33 & 55,0 & 1,21 & $0,96-1,54$ & \\
\hline
\end{tabular}

(continua) 


\begin{tabular}{|c|c|c|c|c|c|c|c|}
\hline \multirow[t]{2}{*}{ Variável } & \multirow[t]{2}{*}{$\mathrm{n}$} & \multirow[t]{2}{*}{$\%$} & \multicolumn{2}{|c|}{ Prevalência de não-exame (\%) } & \multirow[t]{2}{*}{$\mathrm{RP}$} & \multirow[t]{2}{*}{ IC95\% } & \multirow[t]{2}{*}{ p-valor } \\
\hline & & & $\mathrm{n}$ & $\%$ & & & \\
\hline Fumo & & & & & & & 0,002 \\
\hline Nunca fumou & 606 & 59,1 & 271 & 44,7 & 1,00 & & \\
\hline Ex-fumante & 189 & 18,4 & 73 & 38,6 & 0,87 & $0,71-1,05$ & \\
\hline Fumante & 231 & 22,5 & 126 & 54,5 & 1,22 & $1,05-1,41$ & \\
\hline Consultas no último ano & & & & & & & 0,06 \\
\hline 5 ou + & 379 & 42,7 & 141 & 37,2 & 1,00 & & \\
\hline $3-4$ & 211 & 23,8 & 77 & 36,5 & 0,98 & $0,79-1,22$ & \\
\hline $1-2$ & 297 & 33,5 & 134 & 45,1 & 1,21 & $1,01-1,45$ & \\
\hline Tipo de serviço de saúde & & & & & & & $<0,001$ \\
\hline Público & 118 & 34,0 & 60 & 50,8 & 1,00 & & \\
\hline Seguro privado & 199 & 57,6 & 50 & 25,1 & 0,49 & $0,36-0,66$ & \\
\hline Privado & 29 & 8,4 & 3 & 10,3 & 0,20 & $0,06-0,60$ & \\
\hline
\end{tabular}

Em relação à prevenção secundária do câncer de mama, o presente estudo apresentou pelo menos duas limitações: a ausência de questões sobre a realização de mamografia que possibilitaria se conhecer a cobertura populacional e a não-inclusão de mulheres de 61 a 69 anos; ambas as restrições obedeceram a fatores logísticos do projeto Condições de Saúde das Mulheres: Estudo de Base Populacional na Região do Vale do Rio dos Sinos.

Mesmo com os avanços das técnicas diagnósticas, o exame clínico de mamas ainda tem seu papel de destaque na prevenção secundária das neoplasias mamárias 1,7,8,16. Por meio de um exame de baixo custo pode-se obter o diagnóstico precoce, elevando a taxa de cura e aumentando a sobrevida das pacientes com câncer de mama 17 .

Como o exame clínico de mamas depende da possibilidade de acesso à assistência médica, no presente estudo foram considerados fatores reconhecidamente de risco para o câncer mamário (idade, classe econômica, número de filhos, história familiar, obesidade, consumo de bebidas alcoólicas, hábito de fumar) 18, como também características determinantes do uso de serviços de saúde (idade, escolaridade, classe econômica, estado civil, cor da pele, presença de doenças crônicas) 19 .

Em termos de comparação, a cobertura encontrada em São Leopoldo foi inferior àquelas encontradas em outra cidade do Rio Grande do Sul. Os estudos de base populacional realizados em Pelotas investigaram a cobertura do exame clínico de mamas e fatores associados 20,21,22. Deve-se ressaltar que os desfechos e os critérios de inclusão diferiram entre os diferentes estudos. Nos estudos realizados em 1992 e 1999, foram incluídas mulheres de 20 a 69 anos, sendo que os percentuais de mulheres não-examinadas clinicamente foram $20,7 \%$ e $48,9 \%$, respectivamente 20,21 . No estudo mais recente realizado em 2002, incluindo 879 mulheres de 40 a 69 anos, o percentual de cobertura em exames realizados foi de $83,3 \%$ (IC95\%: 80,6-85,7) 22 .

A análise deste estudo revelou que o fator determinante para realização de exame foi a classe econômica. As mulheres inseridas nas classes mais baixas apresentaram, nos dois modelos de análises, menor probabilidade de serem examinadas. Quando se realizou a análise apenas entre as mulheres que procuraram serviços de saúde, persistiu o efeito de classe social adicionado à constatação de que as mulheres não-brancas também foram menos examinadas. Deve-se ressaltar que nos estudos realizados em Pelotas também foram constatados achados semelhantes, revelando que as mulheres com menor inserção de classe e nãobrancas também apresentaram menor probabilidade de serem examinadas 20,21,22.

Fatores relacionados ao acesso aos serviços de saúde, o número de consultas médicas e o tipo de serviço não influenciaram a realização do exame. Da mesma forma, a presença de fatores de risco para o câncer de mama não determinou a realização do exame, evidenciando a falta de lógica epidemiológica para a organização dos serviços de saúde. Na verdade, a presença de fatores de risco deveria orientar para o rastreamento oportunístico seletivo das pacientes 8,23 , de forma que a realização do exame clínico de mama deveria contemplar, principalmente, as mulheres em situação de risco de câncer.

Essa consistência nos achados aponta para problemas no acesso e iniqüidades na assistência. Classe econômica influenciou a realização de exames de mama nos dois modelos analisados. 
Análise multivariada por regressão de Poisson da não-realização de exame médico de mama em todas as mulheres. São Leopoldo, Rio Grande do Sul, Brasil, 2003.

\begin{tabular}{|c|c|c|c|}
\hline Variável & $\mathrm{RP}$ & IC95\% & $p$ valor \\
\hline \multicolumn{4}{|l|}{ Idade (anos) } \\
\hline $20-29$ & 1,00 & & \\
\hline $30-39$ & 0,91 & $0,63-1,32$ & \\
\hline $40-49$ & 0,75 & $0,52-1,07$ & \\
\hline $50-60$ & 0,75 & $0,49-1,14$ & \\
\hline Cor da pele & & & 0,12 \\
\hline Branca & 1,00 & & \\
\hline Não-branca & 1,32 & $0,92-1,89$ & \\
\hline Estado civil & & & 0,09 \\
\hline Casada/Em união & 1,00 & & \\
\hline Outros & 1,28 & $0,96-1,69$ & \\
\hline Classe social & & & 0,005 \\
\hline A & 1,00 & & \\
\hline B & 1,64 & $0,80-3,40$ & \\
\hline C & 2,71 & $1,27-5,77$ & \\
\hline$D$ e $E$ & 3,28 & $1,45-7,29$ & \\
\hline Escolaridade (anos) & & & 0,37 \\
\hline 15 ou + & 1,00 & & \\
\hline $12-14$ & 0,85 & $0,47-1,53$ & \\
\hline $8-11$ & 1,30 & $0,67-2,50$ & \\
\hline $5-7$ & 1,14 & $0,59-2,20$ & \\
\hline $0-4$ & 1,19 & $0,60-2,35$ & \\
\hline Gestações * & & & 0,86 \\
\hline Nenhuma & 1,00 & & \\
\hline $1-3$ & 1,32 & $0,49-3,51$ & \\
\hline 4 ou + & 1,29 & $0,42-3,91$ & \\
\hline Filhos* & & & 0,26 \\
\hline Nenhum & 1,00 & & \\
\hline $1-3$ & 0,69 & $0,27-1,78$ & \\
\hline $4 \mathrm{ou}+$ & 1,10 & $0,36-3,37$ & \\
\hline História familiar de câncer de mama * & & & 0,26 \\
\hline Não & 1,00 & & \\
\hline Sim & 0,79 & $0,53-1,19$ & \\
\hline Uso de álcool (g/dia) * & & & 0,04 \\
\hline$<30$ & 1,00 & & \\
\hline$\geq 30$ & 1,82 & $1,04-3,20$ & \\
\hline Fumo * & & & 0,1 \\
\hline Não-fumante & 1,00 & & \\
\hline Ex-fumante & 0,76 & $0,53-1,10$ & \\
\hline Fumante & 1,20 & $0,87-1,66$ & \\
\hline
\end{tabular}

* Ajustados para classe econômica.

Entretanto, no modelo restrito àquelas mulheres que haviam se consultado no ano, cor da pele foi o fator determinante para a realização do exame, ou seja, superadas as diferenças de acesso, as mulheres brancas terão maior probabilidade de serem examinadas.
Nesse sentido, os achados podem contribuir para colocar em prática os princípios de universalidade e eqüidade na atenção da saúde da mulher no sistema, o que significa atender a todos de acordo com suas necessidades, tratando de forma desigual os desiguais. 
Tabela 3

Análise multivariada por regressão de Poisson da não-realização de exame médico de mama entre as mulheres que se consultaram com médico. São Leopoldo, Rio Grande do Sul, Brasil, 2003.

\begin{tabular}{|c|c|c|c|}
\hline Variável & $\mathrm{RP}$ & IC95\% & $p$ valor \\
\hline Idade (anos) & & & 0,56 \\
\hline $20-29$ & 1,00 & & \\
\hline $30-39$ & 0,93 & $0,75-1,14$ & \\
\hline $40-49$ & 0,82 & $0,66-1,01$ & \\
\hline $50-60$ & 0,87 & $0,70-1,11$ & \\
\hline Cor da pele & & & $<0,05$ \\
\hline Branca & 1,00 & & \\
\hline Não-branca & 1,24 & $1,03-1,48$ & \\
\hline Estado civil & & & 0,57 \\
\hline Casada/Em união & 1,00 & & \\
\hline Outros & 1,07 & $0,91-1,25$ & \\
\hline Classe social & & & $<0,0001$ \\
\hline A & 1,00 & & \\
\hline B & 1,86 & $0,97-3,55$ & \\
\hline C & 3,39 & $1,82-6,31$ & \\
\hline$D e E$ & 3,99 & $2,14-7,44$ & \\
\hline Escolaridade (anos) & & & 0,5 \\
\hline 15 ou + & 1,00 & & \\
\hline $12-14$ & 1,02 & $0,67-1,53$ & \\
\hline $8-11$ & 1,32 & $0,86-2,05$ & \\
\hline $5-7$ & 1,26 & $0,82-1,94$ & \\
\hline $0-4$ & 1,31 & $0,85-2,04$ & \\
\hline Gestações * & & & 0,62 \\
\hline Nenhuma & 1,00 & & \\
\hline $1-3$ & 1,44 & $0,84-2,47$ & \\
\hline 4 ou + & 0,48 & $0,80-2,75$ & \\
\hline Filhos * & & & 0,51 \\
\hline Nenhum & 1,00 & & \\
\hline $1-3$ & 0,97 & $0,79-1,20$ & \\
\hline 4 ou + & 1,15 & $0,90-1,48$ & \\
\hline História familiar de câncer de mama * & & & 0,38 \\
\hline Não & 1,00 & & \\
\hline Sim & 0,85 & $0,65-1,13$ & \\
\hline Uso de álcool (g/dia) * & & & 0,33 \\
\hline$<30$ & 1,00 & & \\
\hline$\geq 30$ & 1,24 & $0,92-1,67$ & \\
\hline Fumo * & & & 0,29 \\
\hline Nunca fumou & 1,00 & & \\
\hline Ex-fumante & 0,83 & $0,66-1,05$ & \\
\hline Fumante & 1,08 & $0,90-1,29$ & \\
\hline Consultas no último ano * & & & 0,68 \\
\hline $5 \mathrm{ou}+$ & 1,00 & & \\
\hline $3-4$ & 0,86 & $0,79-1,20$ & \\
\hline $1-2$ & 1,10 & $0,69-1,75$ & \\
\hline Tipo de serviço de saúde * & & & 0,22 \\
\hline Público & 1,00 & & \\
\hline Seguro privado & 0,76 & $0,50-1,15$ & \\
\hline Privado & 0,41 & $0,12-1,37$ & \\
\hline
\end{tabular}

* Ajustados para classe econômica e cor da pele. 


\section{Resumo}

Foi realizado um estudo transversal de base populacional com o objetivo de estabelecer o nível de cobertura de exame clínico de mamas entre as mulheres de 20 a 60 anos residentes em São Leopoldo, Rio Grande do Sul, Brasil, e descrever as características associadas à sua não-realização. O desfecho foi definido como realização de exame clínico de mamas por médico no ano que antecedeu a entrevista. Foram utilizados dois modelos de análise multivariada. Entre as $1.026 \mathrm{mu}$ lheres incluídas na amostra, 556 (54,2\%; IC95\%: 51,1$57,2)$ foram submetidas ao exame clínico de mamas. A análise multivariada incluindo todas as mulheres demonstrou que somente a variável classe econômica estava associada com a não-realização de exame médico de mama. No outro modelo constatou-se associação entre classe econômica e cor da pele. Assim, classe econômica influenciou a realização de exames de mama nos modelos. Entretanto, no modelo restrito àquelas mulheres que haviam se consultado no ano, cor da pele foi determinante para a realização do exame, ou seja, superadas as diferenças de acesso, as mulheres brancas terão maior probabilidade de serem examinadas.

Exame Físico; Mama; Saúde da Mulher; Desigualdades em Saúde

\section{Referências}

1. Instituto Nacional de Câncer. Programa nacional de controle do câncer do colo do útero e de mama - Viva Mulher. http://www.inca.gov.br (acessado em 06/Dez/2005).

2. Núcleo de Informações em Saúde, Secretaria da Saúde do Rio Grande do Sul. Estatísticas de saúde: mortalidade 2002. Porto Alegre: Secretaria da Saúde do Rio Grande do Sul; 2003.

3. Instituto Nacional de Câncer. Câncer no Brasil: dados dos registros de base populacional. http:// www.inca.gov.br/regpop/2003/ (acessado em 20/ Dez/2006).

4. Olinto MTA, Dias-da-Costa JS, Mendes KG. A saúde em São Leopoldo. Relatório 2000. São Leopoldo: Universidade do Vale do Rio dos Sinos; 2003.

5. Legg JS. The clinical breast exam: opportunities for R.T.s. Radiol Technol 2002; 74:111-22.

6. Moss SM, Cuckle H, Evans A, Johns L, Walter M, Bobrow L. Effect of mammographic screening from age 40 years on breast cancer mortality at 10 years follow-up: a randomized controlled trial. Lancet 2006; 368:2053-60.

\section{Colaboradores}

Todos os autores colaboraram na revisão bibliográfica, análise e redação do artigo.

\section{Agradecimentos}

Financiado pela Fundação de Amparo à Pesquisa do Estado do Rio Grande do Sul (FAPERGS) via Proad2 $\left(n^{\circ} .02 / 0645.9\right)$ e Edital Universal 6 do Conselho de Desenvolvimento Científico e Tecnológico (CNPq, ${ }^{\circ}$. 473478/200-0), com o apoio da Secretaria Municipal de Saúde de São Leopoldo.
7. Shen Y, Parmigiani G. A model-based comparison of breast cancer screening strategies: mammograms and clinical breast examinations. Cancer Epidemiol Biomarkers Prev 2005; 14:529-32.

8. Instituto Nacional de Câncer. Prevenção e controle de câncer. Rev Bras Cancerol 2002; 48:317-32.

9. Dias-da-Costa JS, Olinto MTA, Gigante DP, Menezes AMB, Macedo S, Borba AT, et al. Cobertura do exame citopatológico na cidade de Pelotas, Rio Grande do Sul, Brasil. Cad Saúde Pública 2003; 19:191-7.

10. Associação Nacional de Empresas de Pesquisa. Critério de classificação econômica Brasil. http:// www.anep.org.br (acessado em 13/Fev/2004).

11. Chobanian AV, Bakris GL, Black HR, Cushman WC, Green LA, Izzo Jr. JL, et al. The Seventh Report of the Joint National Committee on Prevention, Detection, Evaluation and Treatment of High Blood Pressure: the JCN 7 report. JAMA 2003; 289:256072 
12. World Health Organization. Obesity: preventing and managing the global epidemic. Report of a WHO Consultation on Obesity. Geneva: World Health Organization; 1998.

13. Moreira LB, Fuchs FD, Moraes RS, Bredemeier M, Cardozo S, Fuchs SC, et al. Alcoholic beverage consumption and associated factors in Porto Alegre, a southern Brazilian city: a population-based survey. J Stud Alcohol 1996; 57:253-9.

14. Victora CG, Huttly SR, Fuchs SC, Olinto MTA. The role of conceptual frameworks in epidemiological analysis: a hierarchical approach. Int J Epidemiol 1997; 26:224-47.

15. Abramson JH. Cross-sectional studies. In: Detels R, Holand WW, McEwen J, Omenn GS, editors. Oxford textbook of public health. New York: Oxford University Press; 1997. p. 517-35.

16. Humphrey LL, Helfand M, Chan BK, Wood SH. Breast cancer screening: a summary of the evidence for the U.S. Preventive Services Task Force. Ann Intern Med 2002; 137(5 Part 1):305-12.

17. Harris JR, Lippman ME, Veronesi U, Willet W. Breast cancer (first of three parts). N Engl J Med 1992; 327:319-28.
18. Instituto Nacional de Câncer. Vigilância do câncer e fatores de risco. http://www.inca.gov.br/vigilancia/ (acessado em 07/Dez/2005).

19. Mendoza-Sassi R, Béria JU. Utilización de los servicios de salud: una revisión sistemática sobre los factores relacionados. Cad Saúde Pública 2001; 17:81932.

20. Dias-da-Costa JS, Piccini RX, Moreira MR. Avaliação da prática do auto-exame e exame físico de mamas na Cidade de Pelotas-RS. Rev Bras Ginecol Obstet 1995; 17:621-32.

21. Dias-da-Costa JS, Olinto MTA, Gigante DP, Menezes AMB, Macedo S, Fuchs SC. Cobertura do exame físico de mama: estudo de base populacional em Pelotas, RS. Rev Bras Epidemiol 2003; 6:39-48.

22. Sclowitz ML, Menezes AMB, Gigante DP, Tessaro S. Condutas na prevenção secundária do câncer de mama e fatores associados. Rev Saúde Pública 2005; 39:340-9.

23. Hutchinson B, Birch S, Evans CE, Goldsmith LJ, Markham BA, Frank J, et al. Selective opportunistic screening for hypercholesterolemia in primary care practice. J Clin Epidemiol 1998; 51:817-25.

Recebido em 12/Jun/2006

Versão final reapresentada em 08/Jan/2007

Aprovado em 18/Jan/2007 